\title{
Direct Electromagnetic Structure Observation by Aberration-corrected Differential Phase Contrast Scanning Transmission Electron Microscopy
}

N. Shibata ${ }^{1}$, S.D. Findlay ${ }^{2}$, T. Matsumoto ${ }^{1}$, T. Seki ${ }^{1}$, G. Sánchez-Santolino ${ }^{1}$, Y. Kohno ${ }^{3}$, H. Sawada ${ }^{3}$, H. Sasaki $^{4}$, Y.G. So ${ }^{5}$, R. Ishikawa ${ }^{1}$ and Y. Ikuhara ${ }^{1,6}$

1. Institute of Engineering Innovation, The University of Tokyo, Tokyo, Japan

2. School of Physics and Astronomy, Monash University, Victoria, Australia

3. JEOL Ltd., Tokyo, Japan

4. Yokohama R\&D Lab., Furukawa Electric Ltd., Yokohama, Japan

${ }^{5 .}$ Department of Materials Science and Engineering, Graduate School of Engineering and Resource Science, Akita University, Akita, Japan

${ }^{6 .}$ Nanostructures Research Laboratory, Japan Fine Ceramic Center, Nagoya, Japan

Electromagnetic structures inside materials, especially at interface regions, critically determine the functional properties of materials and devices because such structures are strongly associated with the dynamics of electrons and holes inside materials. To fundamentally understand the origin of many exotic properties found at interfaces, it is essential to directly characterize local electromagnetic structures even down to atomic dimensions. In recent years, rapid progress in the development of highspeed area detectors for scanning transmission electron microscopy (STEM) has opened new possibilities to image such structures directly at very high-resolution.

In our group, Segmented Annular All-Field (SAAF) detectors, which divide the detector plane into 16 segments and are suitable for use in atomic-resolution STEM imaging [1], have been successfully developed and are now being applied to characterize many materials. In particular, differential phase contrast (DPC) imaging in STEM [2-4] using the SAAF detector can visualize the local electromagnetic field at each raster position and is proving to be very powerful for characterization in real materials and devices. For example, STEM DPC can clearly visualize the strong local electric field variation due to the abrupt potential change within a p-n junction in a GaAs semiconductor (Fig. 1(a)), which cannot be observed by normal annular type dark-field imaging mode (Fig. 1(b)) [5]. Imaging of magnetic structures, such as skyrmions, is also possible using STEM DPC under the objective lens off condition [6], as shown in Fig. 2. Moreover, real-time imaging of electromagnetic structures can now be realized through very fast data processing and reconstruction algorithms based on segmented-type detectors [7]. This real-time imaging can also be applied in atomic-resolution mode, giving interesting and important insights into local electromagnetic structures at interfaces and defects.

In this talk, the current status of the development of the detector and data analysis methods will be reported, along with some applications in materials studies. New prospects for atomic-resolution DPC imaging [8] for materials characterization will be also discussed [9].

References:

[1] N. Shibata et al., J. Electron Microscopy, 59, 473-479 (2010).

[2] N.H. Dekkers and H. de Lang, Optik, 41, 452 (1974).

[3] H. Rose, Ultramicroscopy, 2, 251 (1977). 
[4] W.C. Stewart, J. Opt. Soc. Am., 66, 813 (1976).

[5] N. Shibata et al., Sci. Rep., 5, 10040 (2015).

[6] T. Matsumoto et al., Sci. Adv, 2. e1501280 (2016).

[7] R. Close et al., Ultramicroscopy, 159, 124-137 (2015).

[8] N. Shibata et al., Nature Phys., 8, 611-615 (2012).

[9] This work was supported by the PRESTO and SENTAN, JST and the JSPS KAKENHI Grant number 26289234. A part of this work was supported by Grant-in-Aid for Scientific Research on Innovative Areas (25106003). A part of this work was conducted in Research Hub for Advanced Nano Characterization, The University of Tokyo, under the support of "Nanotechnology Platform" (project No.12024046) by MEXT, Japan. This research was supported under the Discovery Projects funding scheme of the Australian Research Council (Projects No. DP110101570 and DP160102338).


Figure 1. Simultaneous (a) electric field map reconstructed from DPC and (b) ADF STEM image of a p-n junction in a GaAs semiconductor material [5].
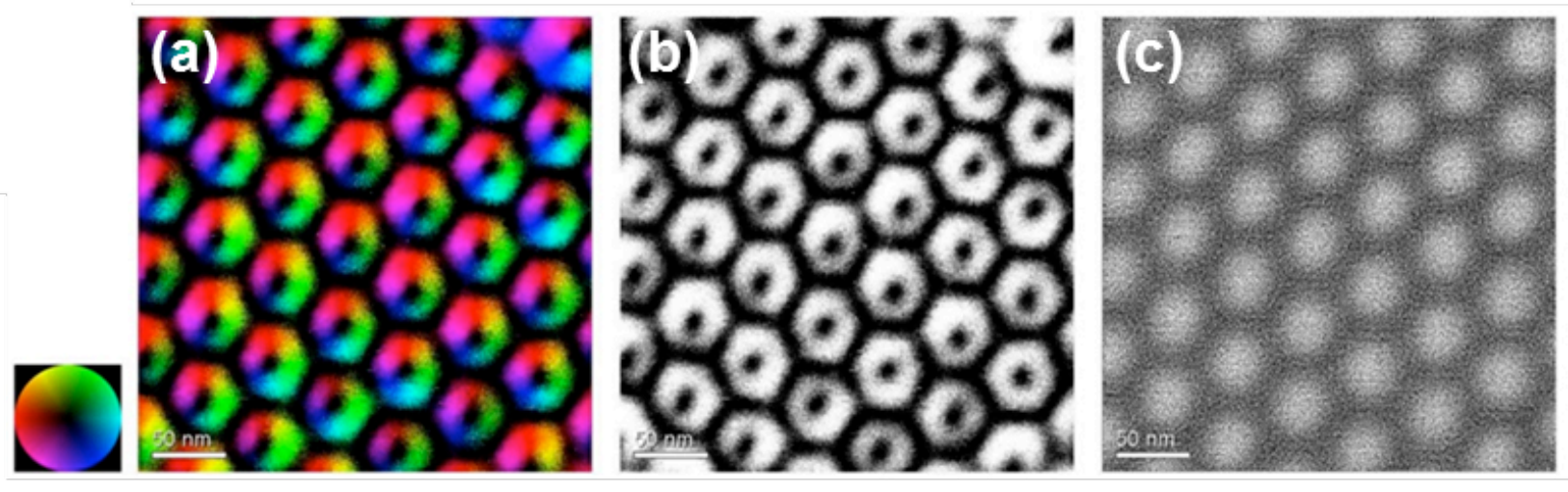

Figure 2. Simultaneous (a) magnetic field map reconstructed from DPC, (b) magnetic field intensity map and (c) helicity image of magnetic skyrmions in $\mathrm{FeGe}_{1-\mathrm{x}} \mathrm{Si}_{\mathrm{x}}[6]$. 